\title{
Verhaltenstherapie
}

\section{Band 15, Heft 4, Dezember 2005}

\section{Schwerpunkt: Schlafstörungen}

Editorial

208 Schlafstörungen - mehr als eine Volkskrankheit Riemann, D. (Freiburg i.Br.)

Übersichtsarbeiten

212 Grundlegendes Wissen über den Schlaf Zulley, J.; Hajak, G. (Regensburg)

220 Diagnostik von Schlafstörungen Weeß, H.-G. (Klingenmünster)

234 Schlaf, Depression und insomnische Beschwerden Riemann, D.; Kühnel, A.; Freyer, T.; Hornyak, M.; Voderholzer, U. (Freiburg i.Br.)

244 Trauma und Schlaf: Ein Überblick Bader, K.; Schäfer, V. (Basel)

Originalarbeit

254 Mit einem «Warum» im Herzen lässt sich fast jedes «Wie» ertragen: Psychotherapeutische Entschlussförderungsintervention Margraf, M. (Lübeck); Berking, M. (Bern)

Interview

262 Rainer Richter: «Eine verfahrensspezifische Ausbildung ist auch weiterhin sinnvoll» Richter, R. (Hamburg)
Vol. 15, Issue 4, December 2005

Focus: Sleep Disorders

Editorial

208 Sleep Disorders - More than a Widespread Disease Riemann, D. (Freiburg i.Br.)

Review Articles

212 Basics about Sleep

Zulley, J.; Hajak, G. (Regensburg)

220 Diagnostics of Sleep Disorders Weeß, H.-G. (Klingenmünster)

234 Sleep, Depression and Insomniac Complaints Riemann, D.; Kühnel, A.; Freyer, T.; Hornyak, M.; Voderholzer, U. (Freiburg i.Br.)

244 Trauma and Sleep: A Review Bader, K.; Schäfer, V. (Basel)

Original Article

254 With a 'Why' in Your Heart Any 'How' Becomes Bearable: A Decision Enhancing Intervention Margraf, M. (Lübeck); Berking, M. (Bern)

Interview

262 Rainer Richter: 'Intervention-Specific Education Will Continue to Make Sense'

Richter, R. (Hamburg)

\section{KARGER}

Fax +49761 4520714

E-mail Information@Karger.de www.karger.com

\section{(C) 2005 S. Karger GmbH, Freiburg}




\section{Verhaltenstherapie}

\section{Band 15, Heft 4, Dezember 2005}

264 Dank an die Gutachter

265 Neuigkeiten vom Buch- und Zeitschriftenmarkt

269 Fort- und Weiterbildung

273 Mitteilungen der Verbände

277 Tagungen und Kongresse

279 Autorenverzeichnis Band 15, 2005

280 Sachwortverzeichnis Band 15, 2005

III Inhalt Band 15, 2005 (nach Seite 282)

II Impressum

U2 Erläuterungen zum Titelbild (2. Umschlagseite) Leibl, C.; Vogt, B.; Rott, A. (Prien)

Einen Ausblick auf den Inhalt der kommenden Hefte finden Sie auf Seite 282.

\section{Vol. 15, Issue 4, December 2005}

264 Acknowledgement to the Reviewers

265 Articles and Books

269 Education

273 Information by Behavior Therapy Associations

277 Meetings and Conferences

279 Author Index Vol. 15, 2005

281 Subject Index Vol. 15. 2005

V Contents Vol. 15, 2005 (following page 282)

II Imprint

C2 Explanations on the Title (Inside front cover) Leibl, C.; Vogt, B.; Rott, A. (Prien)

Forthcoming papers are listed on page 282.

\section{KARGER}

Fax +49761 4520714

E-mail Information@Karger.de

www.karger.com (c) 2005 S. Karger GmbH, Freiburg

Artikel (Volltext) und Inhaltsverzeichnisse

sowie das vorläufige Inhaltsverzeichnis des nächsten Heftes: www.karger.com/ver_bk.htm
NEW

submit your manuser wWW.karger 